\title{
Triangle Centers Associated with Rhombi
}

Floor van Lamoen

Floor van Lamoen was born in 1966 in Leiden (NL). He studied mathematics at the University of Amsterdam and graduated in 1990. After his study he chose to become a high school teacher, his current profession. It was also after his study that he was attracted to the beauty of plane Euclidean and related geometry, and more specifically triangle geometry. Outside mathematics he is a national level racewalker. $\mathrm{He}$ is married and has one daughter.

\begin{abstract}
Not many of the famous triangle centers, or other central objects, are associated with rhombi, or even squares. In this paper four examples of such centers are given. They are related to well-known central objects in a triangle.
\end{abstract}

\section{Introduction}

Triangle geometry usually is about triangles, lines, circles, conic sections and cubic curves. Quadrilaterals do not to come into the picture very often. We can use rhombi however quite well to define pleasant triangle centers.

In the representation of triangle centers, we will make use of homogeneous barycentric coordinates, or shortly barycentrics, with respect to a fixed reference triangle $A B C$. The notion of these coordinates goes back to Möbius. Traditionally the point with coordinates $(\xi, \eta, \zeta)$ is considered as the center of mass (barycenter) of $\triangle A B C$ when located in

Wer glaubt, die Dreiecksgeometrie sei ein abgeschlossenes Gebiet, den lehrt dieser Beitrag, dass auch heute noch Neues und dabei auch Interessantes in der Elementargeometrie entdeckt werden kann - und das in der traditionellen Art und Weise, also ohne Einsatz eines Computers, d.h. ohne dynamische Geometriesoftware.

Im Falle ausgezeichneter Punkte eines Dreiecks haben wir den Umkreis- und Inkreismittelpunkt, den Höhenschnittpunkt (Orthozentrum) und den Schwerpunkt (Baryzentrum) noch aus der Schule in Erinnerung. In dem vorliegenden Beitrag nutzt F. van Lamoen die von A.F. Möbius eingeführten baryzentrischen Koordinaten bzw. trilinearen Koordinaten und stösst dabei auf interessante Rhomben und Quadrate, die sich so als ausgezeichnete Figuren des Dreiecks erweisen. $j k$ 
$A, B$ and $C$ are 'weights' of magnitudes $\xi, \eta$ and $\zeta$ respectively (and the rest of the triangle is weightless).

More conveniently the equivalent definition is used, that $(\xi, \eta, \zeta)$ denotes the point $P$ such that $\operatorname{Area}(P B C): \operatorname{Area}(A P C): \operatorname{Area}(A B P)=\xi: \eta: \zeta$. Here, we take $\operatorname{Area}(P Q R)$ to be zero when $P, Q$ and $R$ are collinear, positive when the orientation of the vertices of $\triangle P Q R$ is counter-clockwise, and negative when the orientation is clockwise.

Barycentric coordinates are homogeneous in the sense that for any nonzero real number $t$ the coordinates $(\xi, \eta, \zeta)$ and $(t \xi, t \eta, t \zeta)$ refer to the same point. For this reason we will write $(\xi: \eta: \zeta)$.

All coordinates in this paper will be barycentrics, unless mentioned otherwise.

Rather similar to barycentric coordinates is the notion of trilinear coordinates (trilinears). Trilinear coordinates $(\xi: \eta: \zeta)$ denote the point $P$ with ratio of distances to the sidelines $d\left(P, \ell_{B C}\right): d\left(P, \ell_{A C}\right): d\left(P, \ell_{A B}\right)=\xi: \eta: \zeta\left(\ell_{X Y}\right.$ denotes the line through $X$ and $\left.Y\right)$. We give sign to, for instance, $d\left(P, \ell_{A B}\right)$ in the same way as to $\operatorname{Area}(A B P)$ in barycentric coordinates. Trilinear coordinates are homogeneous in the same sense as barycentrics. They are used and explained extensively in [2].

A simple relation between barycentric coordinates $\left(\xi_{b}: \eta_{b}: \zeta_{b}\right)$ and trilinear coordinates $\left(\xi_{t}: \eta_{t}: \zeta_{t}\right)$ for the same point $P$ is

$$
\xi_{b}: \eta_{b}: \zeta_{b}=a \xi_{t}: b \eta_{t}: c \zeta_{t}=\xi_{t} \sin \alpha: \eta_{t} \sin \beta: \zeta_{t} \sin \gamma,
$$

where $a=|B C|, b=|A C|, c=|A B|, \alpha=\angle A, \beta=\angle B$ and $\gamma=\angle C$.

In both coordinate systems a line $\ell$ is given by the equation $l x+m y+n z=0$. We usually write this as $\ell=[l: m: n]$, and observe that these line coordinates are homogeneous. Treating lines, as well as points, as vectors we find the line $\ell_{P_{1} P_{2}}$ as external product $\ell=P_{1} \times P_{2}$. Dually, the point of intersection $P$ of lines $\ell_{1}$ and $\ell_{2}$ is found by $P=\ell_{1} \times \ell_{2}$.

A notion from triangle geometry that we will use several times in this paper is isogonal conjugacy. Two points $P$ and $Q$ are called isogonal conjugates when $\angle B A P=\angle Q A C$, $\angle C B P=\angle Q B A$ and $\angle A C P=\angle Q C B$. Algebraicly the isogonal conjugate of $P=(\xi$ : $\eta: \zeta)$, not on the sidelines of $\triangle A B C$, is $Q=\left(a^{2} / \xi: b^{2} / \eta: c^{2} / \zeta\right)$.

In this paper four ways will be presented to define triangle centers associated with rhombi. Points $\mathscr{A}_{\psi}, \mathscr{B}_{\psi}, \mathscr{C}_{\psi}$ and $\mathscr{D}_{\psi}$ will be (triangle) centers following the definition by Clark Kimberling in [2].

Kimberling's notion of center depends on the definition of center functions. Let $f(a, b, c)$ be a nonzero continuous function defined on triples $(a, b, c)$, representing the sidelengths of the reference triangle. Function $f$ is a center function

- if $f$ is homogeneous, i.e. $\exists r \forall k: f(k a, k b, k c)=k^{r} f(a, b, c)$;

- and if $f$ is symmetric in the sense that $f(a, b, c)=f(a, c, b)$.

Any point of the form $(f(a, b, c): f(b, c, a): f(c, a, b))$ (either in trilinears, or in barycentrics) is called a center. Hereby a center is not a point in the classical sense, but it is seen as a function of the triangle it is defined in. 
The first rhombi-center $\mathscr{A}_{\psi}$ will be associated with rhombi circumscribing $\triangle A B C, \mathscr{C}_{\psi}$ with rhombi inscribed in $\triangle A B C, \mathscr{B}_{\psi}$ and $\mathscr{D}_{\psi}$ will be defined by three congruent rhombi. The centers will be depending on the internal angles $\psi$ and $\bar{\psi}:=\pi-\psi$ of the rhombi. Here $\psi(A, B, C)$ is considered to be independent from permutation of $(A, B, C)$.

Applying more of Clark Kimberling's definitions, centers $\mathscr{A}_{\psi}$ and $\mathscr{B}_{\psi}$ for fixed $\psi$ will be major centers (see [1]), since these centers can be written as $(f(\alpha): f(\beta): f(\gamma))$ for some continuous function $f$. Finally, $\triangle A^{\prime} B^{\prime} C^{\prime}$ of Section 2 will be a central triangle (see [3] for the least complicated definition).

\section{Rhombi circumscribing a triangle}

The definition of the first triangle center $\mathscr{A}_{\psi}$ depends on the following:

Theorem 1 Let $\triangle A B C$ and $\psi \in(-\pi, \pi) \backslash\{0\}$ be given. We can construct a unique rhombus $A X A^{\prime} Y$ such that $\angle X A Y=\psi, B \in \ell_{X A^{\prime}}$ and $C \in \ell_{A^{\prime} Y}$. Similarly we can construct rhombi to find points $B^{\prime}$ and $C^{\prime}$. The constructed $\triangle A^{\prime} B^{\prime} C^{\prime}$ is perspective to $\triangle A B C$, i.e. $\ell_{A A^{\prime}}, \ell_{B B^{\prime}}$ and $\ell_{C C^{\prime}}$ are concurrent.

The perspector (center of perspectivity) $\ell_{A A^{\prime}} \cap \ell_{B B^{\prime}} \cap \ell_{C C^{\prime}}$ will be called the circumrhombi point $\mathscr{A}_{\psi}$.

Proof. Denote by $\rho_{\chi, X}(P)$ the image of $P$ after rotation through $\chi$ about $X$. Let $B^{\prime \prime}=$ $\rho_{\bar{\psi}, A}(B)$ and let $C^{\prime \prime}=\rho_{-\bar{\psi}, A}(C)$. Then let $\left\{A^{\prime}\right\}=\ell_{B^{\prime \prime} C} \cap \ell_{C^{\prime \prime} B}$. Points $X \in \ell_{C^{\prime \prime} A^{\prime}}$ and $Y \in \ell_{B^{\prime \prime} A^{\prime}}$ can be constructed in such a way that $A X A^{\prime} Y$ is a parallelogram (see Figure 1).

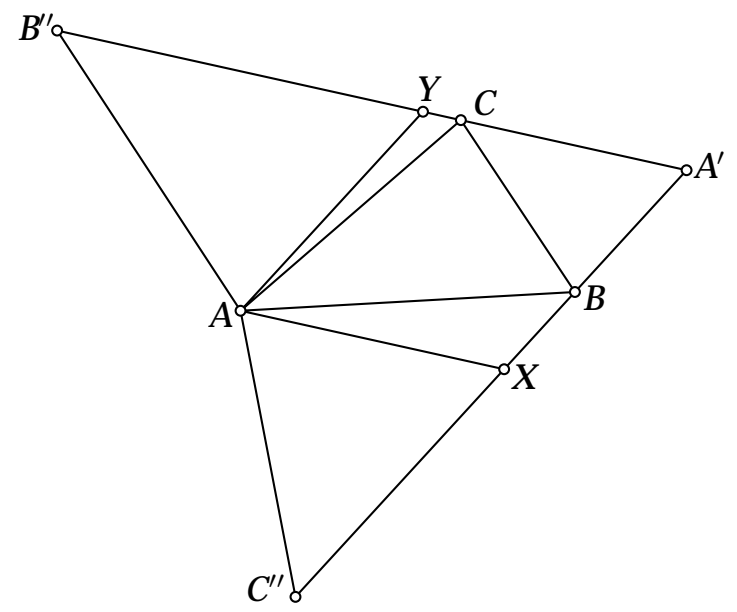

Fig. 1 Construction of rhombus $A X A^{\prime} Y$ circumscribing $\triangle A B C$ for $\psi=\frac{\pi}{3}$.

Observe that $\triangle A C^{\prime \prime} B \cong \triangle A C B^{\prime \prime}$, so the perpendicular distances from $A$ to $\ell_{B^{\prime \prime}} A^{\prime}$ and $\ell_{C^{\prime \prime} A^{\prime}}$ are equal. And $A X A^{\prime} Y$ must be a rhombus. 
Note that $\rho_{\bar{\psi}, A}\left(\ell_{C^{\prime \prime} X B A^{\prime}}\right)=\ell_{A^{\prime} C Y B^{\prime \prime}}$, so $\angle Y A^{\prime} X=\psi$ (regarding orientation). And $A X A^{\prime} Y$ is a rhombus satisfying the requirements of the theorem.

It is easy to see that $A X A^{\prime} Y$ is the unique rhombus fulfilling these requirements: When we rotate the complete figure of $\triangle A B C$ and rhombus $A X A^{\prime} Y$ through $\bar{\psi}$ about $A$, and let $B^{\prime \prime}$ be the image of $B$ again, we immediately see that $B^{\prime \prime} \in \ell_{A^{\prime} C}$. In the same way we see that the image of $C$ after rotation through $-\bar{\psi}$ about $A$ must be on the line $\ell_{A^{\prime} B}$.

To prove the last statement of the theorem, we use barycentrics. First we calculate barycentrics for $C^{\prime \prime}$. To do this, let $C_{a}^{\prime \prime}, C_{b}^{\prime \prime}$ and $C_{c}^{\prime \prime}$ be the orthogonal projections of $C^{\prime \prime}$ on sidelines $\ell_{B C}, \ell_{A C}$ and $\ell_{A B}$ respectively. We will calculate the trilinear distances $\xi:= \pm\left|C^{\prime \prime} C_{a}^{\prime \prime}\right|, \eta:= \pm\left|C^{\prime \prime} C_{b}^{\prime \prime}\right|$ and $\zeta:= \pm\left|C^{\prime \prime} C_{c}^{\prime \prime}\right|$, given signs in the usual way for trilinear coordinates.

Straightforwardly we find that $\eta=b \sin \psi$ and $\zeta=-b \sin (\bar{\psi}-\alpha)=-b \sin (\alpha+\psi)$. Using that $\triangle C^{\prime \prime} A C$ is isosceles, we see $\left|C^{\prime \prime} C\right|=2 b \cos \left(\frac{\psi}{2}\right)$, and consequently $\xi=$ $2 b \cos \left(\frac{\psi}{2}\right) \sin \left(\gamma-\frac{\psi}{2}\right)$. With use of (1) this brings as barycentrics for $C^{\prime \prime}$ :

$$
C^{\prime \prime}=\left(2 a \cos \left(\frac{\psi}{2}\right) \sin \left(\gamma-\frac{\psi}{2}\right): b \sin \psi:-c \sin (\alpha+\psi)\right) .
$$

In the same way $B^{\prime \prime}$ becomes:

$$
B^{\prime \prime}=\left(2 a \cos \left(\frac{\psi}{2}\right) \sin \left(\beta-\frac{\psi}{2}\right):-b \sin (\alpha+\psi): c \sin \psi\right) .
$$

Straightforward calculations give that barycentrics for $\left\{A^{\prime}\right\}=\ell_{B B^{\prime \prime}} \cap \ell_{C C^{\prime \prime}}$ are:

$$
A^{\prime}=\left(-2 a \csc (\alpha+\psi): b \sec \left(\frac{\psi}{2}\right) \csc \left(\beta-\frac{\psi}{2}\right): c \sec \left(\frac{\psi}{2}\right) \csc \left(\gamma-\frac{\psi}{2}\right)\right) .
$$

With similar results for $B^{\prime}$ and $C^{\prime}$, we conclude that $\ell_{A A^{\prime}}, \ell_{B B^{\prime}}$ and $\ell_{C C^{\prime}}$ concur in

$$
\mathscr{A}_{\psi}=\left(\sin \alpha \csc \left(\alpha-\frac{\psi}{2}\right): \sin \beta \csc \left(\beta-\frac{\psi}{2}\right): \sin \gamma \csc \left(\gamma-\frac{\psi}{2}\right)\right) \text {. }
$$

All circumrhombi points lie on the Kiepert hyperbola, a rectangular hyperbola consisting of the isogonal conjugates of points on the Brocard axis (the line through the circumcenter and the symmedian or Lemoine point). See also Section 3.

For $\psi= \pm \frac{2 \pi}{3}$ we find that $\triangle A^{\prime} B^{\prime} C^{\prime}$ degenerates into a single point. The two points are the isogonic centers ( $X_{13}$ (the Fermat-Torricelli point) and $X_{14}$ in [2]).

Among the huge list of triangle centers in [2] there are quite a few centers that can be found as circumrhombi points. Examples are the Napoleon points, the third Brocard point and the Tarry point. Points that might be added are the circumsquares points, found when $\psi= \pm \frac{\pi}{2}$. 


\section{Congruent rhombi points}

Let $\psi \in[-\pi, \pi]$. We call $\mathscr{B}_{\psi}$ a congruent rhombi point if there exist six points $P_{A}, Q_{C} \in$ $\ell_{A C}, P_{C}, Q_{B} \in \ell_{B C}$ and $Q_{A}, P_{B} \in \ell_{A B}$ such that:

- These six points are on a circle with center $\mathscr{B}_{\psi}$;

- $\angle P_{A} \mathscr{B}_{\psi} Q_{A}=\angle P_{B} \mathscr{B}_{\psi} Q_{B}=\angle P_{C} \mathscr{B}_{\psi} Q_{C}=\psi$.

Triangles $P_{A} \mathscr{P}_{\psi} Q_{A}, P_{B} \mathscr{B}_{\psi} Q_{B}$ and $P_{C} \mathscr{B}_{\psi} Q_{C}$ are congruent and form the halves of three congruent rhombi: $P_{A} \mathscr{P}_{\psi} Q_{A} A^{\prime}$ (the A-rhombus), $P_{B} \mathscr{P}_{\psi} Q_{B} B^{\prime}$ and $P_{C} \mathscr{B}_{\psi} Q_{C} C^{\prime}$ (see Figure 2).

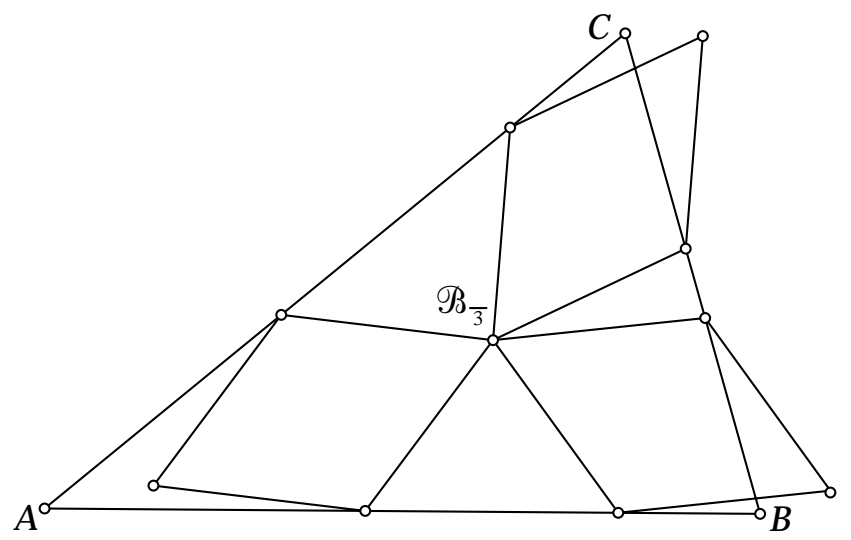

Fig. 2 The congruent rhombi point $\Re_{\frac{\pi}{3}}$.

This way to define a triangle center is inspired by the Kenmotu point or congruent squares point, found in Kimberling's list of triangle centers in [2] as $X_{371}$, which point is $\mathscr{B}_{\frac{\pi}{2}}$. The Kenmotu point, named after the author of a Japanese mathematics problem collection in 1840 , is the only center in this list, which is given a description directly using squares or rhombi. The point $\mathscr{B}_{-\frac{\pi}{2}}$ is mentioned in [2] as $X_{372}$, but without definition using squares directly.

Theorem 2 The congruent rhombi point $\mathscr{B}_{\psi}$ is the point with barycentrics

$$
\left(\sin \alpha \cos \left(\alpha-\frac{\psi}{2}\right): \sin \beta \cos \left(\beta-\frac{\psi}{2}\right): \sin \gamma \cos \left(\gamma-\frac{\psi}{2}\right)\right) .
$$

Proof. Suppose the point $\mathscr{B}_{\psi}$ and the points $P_{A}, P_{B}, P_{C}, Q_{A}, Q_{B}$ and $Q_{C}$ from the definition exist. Let $\chi_{a}:=\angle Q_{B} \mathscr{P}_{\psi} P_{C}, \chi_{b}:=\angle Q_{C} \mathscr{P}_{\psi} P_{A}$ and $\chi_{c}:=\angle Q_{A} \mathscr{P}_{\psi} P_{B}$. The circle with $A \mathscr{P}_{\psi}$ as diameter passes through the midpoints of $Q_{A} P_{B}$ and $P_{A} Q_{C}$. From this and similar results for the circles with diameters $B \mathscr{B}_{\psi}$ and $C \mathscr{B}_{\psi}$ we find the following system of equations:

$$
\frac{\chi_{a}}{2}+\frac{\chi_{b}}{2}+\psi=\alpha+\beta, \quad \frac{\chi_{b}}{2}+\frac{\chi_{c}}{2}+\psi=\beta+\gamma, \quad \frac{\chi_{a}}{2}+\frac{\chi_{c}}{2}+\psi=\alpha+\gamma .
$$


This gives $\chi_{a}=2 \alpha-\psi, \chi_{b}=2 \beta-\psi$ and $\chi_{c}=2 \gamma-\psi$. Since $\chi_{a}, \chi_{b}$ and $\chi_{c}$ are the top angles of three isosceles triangles on the sides of $\triangle A B C$ with congruent legs, $\mathscr{B}_{\psi}$ must have barycentrics as in the theorem.

On the other hand, we can construct a point $\mathscr{B}_{\psi}$ with barycentrics as in the theorem when $\psi$ is given: $\mathscr{B}_{\psi}$ is the isogonal conjugate of $\mathscr{A}_{-\bar{\psi}}$ when $\psi \in(-\pi, \pi) \backslash\{0\}$, the circumcenter when $\psi=0$ and the symmedian point when $\psi= \pm \pi$. Having $\mathscr{B}_{\psi}$, we can also construct the points $P_{A}, P_{B}, P_{C}, Q_{A}, Q_{B}$ and $Q_{C}$ using the results from the first part of the proof.

All congruent rhombi points $\mathscr{B}_{\psi}$ lie on the Brocard axis. Well-known centers that can be found as $\mathscr{B}_{\psi}$ are the isodynamic points and the Brocard midpoint.

It is not difficult to see that $\triangle A B C$ and $\triangle A^{\prime} B^{\prime} C^{\prime}$ ( $A^{\prime}$ was the vertex of the $A$-inrhombus opposite to $\mathscr{B}_{\psi} ; B^{\prime}$ and $C^{\prime}$ were found similarly) are homothetic (parallel). Let $\xi$ be the perpendicular distance between $B C$ and $B^{\prime} C^{\prime}$, given sign in the usual way for trilinear coordinates w.r.t. $\triangle A B C$, and let $\eta$ and $\zeta$ be defined likewise. Then straightforward computation gives us that

$$
\xi: \eta: \zeta=\cos \left(\alpha+\frac{\psi}{2}\right): \cos \left(\beta+\frac{\psi}{2}\right): \cos \left(\gamma+\frac{\psi}{2}\right) .
$$

We can apply the following lemma:

Lemma 3 Let $\triangle A B C$ and $\triangle A^{\prime} B^{\prime} C^{\prime}$ be homothetic. Let $\xi$, $\eta$ and $\zeta$ be the distances between the $a$-, $b$-and c-sides of these triangles respectively, given signs in the usual way for trilinear coordinates w.r.t. $\triangle A B C$. Then the perspector of $\triangle A B C$ and $\triangle A^{\prime} B^{\prime} C^{\prime}$ has trilinears $(\xi: \eta: \zeta)$ w.r.t. both triangles.

The proof of this lemma is easy and left to the reader.

Using Lemma 3 we find that the perspector of $\triangle A B C$ and $\triangle A^{\prime} B^{\prime} C^{\prime}$ is $\mathscr{B}_{-\psi}$.

Consequently, but this is also seen directly from angle computations using earlier results on the top angles of triangles such as $\triangle Q_{C} \mathscr{B}_{\psi} P_{A}$, each pair of $A$-, $B$ - and $C$-rhombi for $\psi$ and $-\psi$ must be parallel. See Figure 3 .

\section{Inrhombi points}

The easiest way to find a triangle center using rhombi seems to be the use of rhombi inscribed in $\triangle A B C$.

Given $\triangle A B C$, we can compute the sidelength of a rhombus TUVW with $\angle T U V=$ $\psi \in\left[-\frac{\pi}{2}, \frac{\pi}{2}\right] \backslash\{0\}$ in such a way that these conditions hold: $\ell_{T U}=\ell_{A B}, V \in \ell_{B C}$, $W \in \ell_{A C}$ and points $V$ and $W$ lie on the same side of $\ell_{A B}$ as $C$. We will call rhombus TUVW the $C$-inrhombus. After similarly having found the $A$ - and $B$-inrhombi, $\ell_{V W}$ is the $c$-side of a $\triangle A^{\prime} B^{\prime} C^{\prime}$ which is homothetic to $\triangle A B C$. The perspector $\mathscr{C}_{\psi}$ of $\triangle A^{\prime} B^{\prime} C^{\prime}$ and $\triangle A B C$ will be called the inrhombi point.

To calculate the sidelengths of TUVW let $p:=|V W| / c=|V C| / a=|W C| / b$. We find that:

$$
\begin{aligned}
|V W| & =p c, \\
|V U| & =\frac{(1-p) a \sin \beta}{\sin \psi} .
\end{aligned}
$$




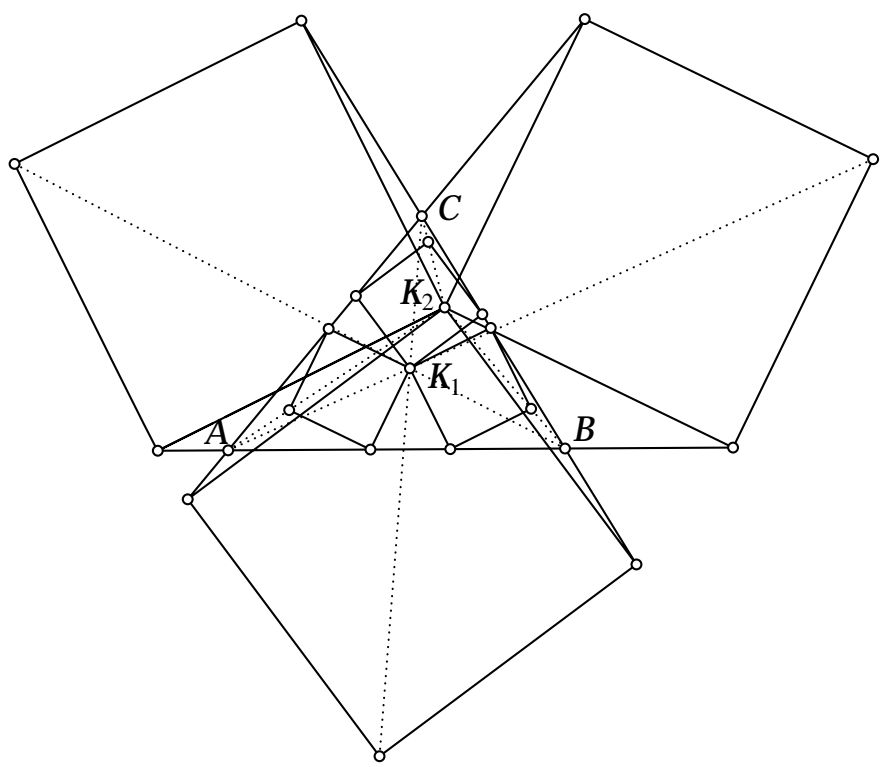

Fig. 3 Parallel squares and the two congruent squares points $K_{1}$ and $K_{2}$.

Then $(2)=(3)$ gives

$$
p=\frac{a \sin \beta}{a \sin \beta+c \sin \psi} .
$$

Substituting (4) into (2) we see that

$$
|V W|=\frac{2 \sigma}{a \sin \beta+c \sin \psi},
$$

where $\sigma$ denotes the area of $\triangle A B C$. Achieving similar results for $A$ - and $B$-inrhombi, and using Lemma 3 , we conclude that barycentrics for $\mathscr{C}_{\psi}$ are

$$
\left(\frac{\sin \alpha}{\sin \beta \sin \gamma+\sin \alpha \sin \psi}: \frac{\sin \beta}{\sin \alpha \sin \gamma+\sin \beta \sin \psi}: \frac{\sin \gamma}{\sin \alpha \sin \beta+\sin \gamma \sin \psi}\right) .
$$

All inrhombi points lie on the hyperbola formed by the isogonal conjugates of points on the line through the centroid and the symmedian point of $\triangle A B C$.

\section{Congruent shrinked inrhombi points}

For $\psi \in\left[-\frac{\pi}{2}, \frac{\pi}{2}\right]$, let $B_{A}, A_{B} \in \ell_{A B}, A_{C}, C_{A} \in \ell_{A C}, B_{C}, C_{B} \in \ell_{B C}$ and $\mathscr{D}_{\psi}$ fulfil the following statements:

- $\ell_{B_{A} C_{A}}\left\|\ell_{B C}, \ell_{A_{B} C_{B}}\right\| \ell_{A C}$ and $\ell_{A_{C} B_{C}} \| \ell_{A B}$

- $\ell_{B_{A} C_{A}} \cap \ell_{A_{B} C_{B}} \cap \ell_{A_{C} B_{C}}=\left\{\mathscr{D}_{\psi}\right\}$

- The $A$-inrhombus of $\triangle A B_{A} C_{A}$, the $B$-inrhombus of $\triangle A_{B} B C_{B}$ and the $C$-inrhombus of $\triangle A_{C} B_{C} C$ are congruent. 

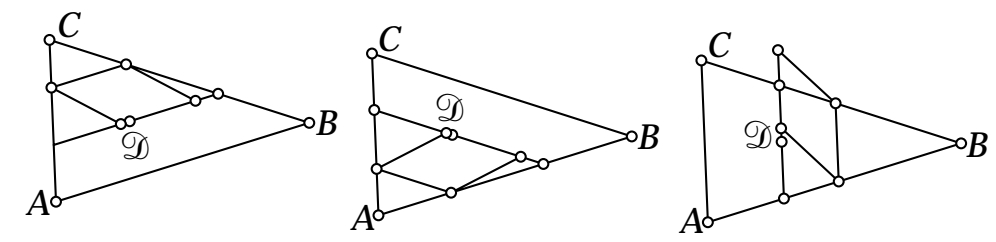

Fig. 4 The congruent shrinked inrhombi point $\mathscr{D}$ for $\psi=\frac{\pi}{4}$.

$\mathscr{D}_{\psi}$ will be called the congruent shrinked inrhombi point. See Figure 4.

To calculate coordinates for (possible) $\mathscr{D}_{\psi}$ let $q_{a}:=\left|B_{A} C_{A}\right| /|B C|$ and let $q_{b}$ and $q_{c}$ be defined likewise. To make the shrinked inrhombi congruent, we derive from (5) and similar results on sidelengths of the $A$ - and $B$-inrhombi that there must be a real number $t$, such that $q_{a}=t(b \sin \gamma+a \sin \psi), q_{b}=t(a \sin \gamma+b \sin \psi)$ and $q_{c}=t(a \sin \beta+b \sin \psi)$.

It is also easy to see that $\operatorname{Area}\left(\triangle B C \mathscr{D}_{\psi}\right)=\left(1-q_{a}\right) \sigma$, Area $\left(\triangle A C \mathscr{D}_{\psi}\right)=\left(1-q_{b}\right) \sigma$ and $\operatorname{Area}\left(\triangle A B \mathscr{D}_{\psi}\right)=\left(1-q_{c}\right) \sigma$. The sum of these three areas is $\sigma$, so $q_{a}+q_{b}+q_{c}=2$ and consequently

$$
t=\frac{2}{b \sin \gamma+a \sin \gamma+a \sin \beta+(a+b+c) \sin \psi} .
$$

And we find that $\mathscr{D}_{\psi}$ has barycentrics

$$
\begin{gathered}
(\sin \alpha(\sin \beta+\sin \gamma)-\sin \beta \sin \gamma+\sin \psi(\sin \beta+\sin \gamma-\sin \alpha): \\
\sin \beta(\sin \alpha+\sin \gamma)-\sin \alpha \sin \gamma+\sin \psi(\sin \alpha+\sin \gamma-\sin \beta): \\
\sin \gamma(\sin \alpha+\sin \beta)-\sin \alpha \sin \beta+\sin \psi(\sin \alpha+\sin \beta-\sin \gamma))
\end{gathered}
$$

The congruent shrinked inrhombi points all lie on the line through the Nagel point and the equal parallelians point ( $X_{192}$ in [2], found when $\psi=0$ ). Only four centers on this line are mentioned in [2]. The congruent shrinked insquares points might be interesting additions to these.

\section{References}

[1] Clark Kimberling, Major Centers of Triangles, Amer. Math. Monthly, 104 431-438 (1997).

[2] Clark Kimberling, Triangle Centers and Central Triangles, Congressus Numerantium, 129 (1998).

[3] Clark Kimberling and Floor van Lamoen, Central Triangles, Nieuw Arch. Wisk., 17 1-19 (1999).

Floor van Lamoen

Statenhof 3

4463 TV Goes

The Netherlands

e-mail: f.v.lamoen@wxs.nl 